\title{
Venetoclax and BCR-ABL Tyrosine Kinase Inhibitor Combinations: Outcome in Patients with Philadelphia Chromosome-Positive Advanced Myeloid Leukemias
}

\author{
Abhishek Maiti ${ }^{a}$ b Miguel J. Franquiz ${ }^{a}$ Farhad Ravandi ${ }^{a}$ Jorge E. Cortes ${ }^{a}$ \\ Elias J. Jabbour ${ }^{\mathrm{a}}$ Koji Sasaki ${ }^{\mathrm{a}}$ Kayleigh Marx ${ }^{\mathrm{a}}$ Naval G. Daver ${ }^{\mathrm{a}}$ \\ Tapan M. Kadia ${ }^{a}$ Marina Y. Konopleva ${ }^{a}$ Lucia Masarova $^{a}$ Gautam Borthakura \\ Courtney D. DiNardo ${ }^{a}$ Kiran Naqvi $^{a}$ Sherry Pierce ${ }^{a}$ Hagop M. Kantarjian $^{a}$ \\ Nicholas J. Short ${ }^{a}$ \\ a Department of Leukemia, The University of Texas MD Anderson Cancer Center, Houston, TX, USA; \\ ${ }^{b}$ Division of Cancer Medicine, The University of Texas MD Anderson Cancer Center, Houston, TX, USA
}

\section{Keywords \\ Venetoclax · Acute myeloid leukemia $\cdot B C R / A B L \cdot$ Chronic myeloid leukemia - Decitabine · Ponatinib - Tyrosine kinase inhibitors $\cdot$ Myeloid blast phase $\cdot$ Philadelphia chromosome-positive acute myeloid leukemia}

\begin{abstract}
Background: Philadelphia chromosome-positive $(\mathrm{Ph}+) \mathrm{ad}-$ vanced leukemias, including acute myeloid leukemia (AML) and chronic myeloid leukemia (CML) in myeloid blast phase (MBP), have poor outcomes. Venetoclax has shown synergism with BCR-ABL1 tyrosine kinase inhibitors (TKI) in preclinical studies. However, clinical activity of venetoclax and TKI-based regimens is unknown. Methods: We conducted a retrospective study on patients with $\mathrm{Ph}+\mathrm{AML}(n=7)$ and CML-MBP $(n=9)$ who received venetoclax combined with TKI-based regimens at our institution. Results: Median patient age was 42 years, and the median number of prior therapy cycles was 5 (range 2-8). Nine patients received
\end{abstract}

karger@karger.com www.karger.com/aha

Karger" decitabine-based, and 7 received intensive chemotherapybased regimens. Ten patients (63\%) received ponatinib. The overall response rate (ORR) in 15 evaluable patients was $60 \%$ (1 complete remission [CR], 6 CR with incomplete hematologic recovery [CRi], 1 morphologic leukemia-free state, and 1 partial response). The ORR was $43 \%$ in $\mathrm{Ph}+\mathrm{AML}$ and $75 \%$ in CML-MBP. The median overall survival (OS) for all patients was 3.6 months, for AML OS was 2.0 months, and for CMLMBP OS was 10.9 months. The median relapse-free survival for AML and CML-MBP was 3.6 and 3.9 months, respectively. Compared to nonresponders, patients achieving CR/CRi had higher baseline Ph+ metaphases and BCR-ABL1 PCR. Conclusions: Combination therapy of venetoclax with TKIbased regimens shows encouraging activity in very heavily pretreated, advanced $\mathrm{Ph}+$ leukemias, particularly CML-MBP.

(c) 2020 S. Karger AG, Basel

Part of this study was published online as an abstract in the Proceedings of the American Society of Clinical Oncology Annual Meeting 2019. 


\section{Introduction}

Acute myeloid leukemia (AML) and chronic myeloid leukemia (CML) in myeloid blast phase (MBP) are aggressive myeloid leukemias with overall poor outcomes $[1,2]$. Both AML and CML-MBP are more frequently diagnosed in older patients, with a median age of $>60$ years at diagnosis $[3,4]$. While AML is the most common acute leukemia in adults, CML is much less common, and only a minority of patients progress to the aggressive blast phase. $t(9 ; 22)$ leads to the formation of the Philadelphia chromosome $(\mathrm{Ph})$, which is almost universally present in CML and leads to the development of the constitutively active BCR-ABL tyrosine kinase. However, in rare instances, $\mathrm{Ph}$ has been noted in AML [5-7]. Patients with newly diagnosed $\mathrm{Ph}+\mathrm{AML}$ have a poor outcome, with a reported median overall survival (OS) of 9 months, although there is a dearth of data on this entity given its rarity $[5,6]$. Transformation to advanced-phase CML occurs in $1-5 \%$ patients in chronic phase (CP) CML per year, and CML-MBP has a median OS of 6-12 months despite the use of second- or third-generation tyrosine kinase inhibitors (TKI) $[2,8,9]$. These poor outcomes highlight the need for novel therapies for these patients.

The anti-apoptotic BCL2 family of proteins regulate the mitochondrial apoptotic response. BCL2 is highly and almost universally expressed in leukemia cells and leukemic stem cells in AML and CML, is upregulated by BCRABL1 signaling, and is overexpressed in CML blast phase [10-14]. Venetoclax, a selective BCL2 inhibitor, is approved for use with low-dose cytarabine or hypomethylating agents for older or "unfit" patients with newly diagnosed AML. Venetoclax has shown preclinical activity against TKI-resistant CML cells and has shown synergism with BCR-ABL TKI in eradicating leukemic stem cells in advanced CML $[13,15,16]$. However, clinical activity of venetoclax and BCR-ABL TKI-based combinations is unknown. Hence, we conducted a retrospective study to summarize the activity of such combination therapies in advanced $\mathrm{Ph}+$ myeloid leukemias.

\section{Materials and Methods}

In this retrospective chart review study, we included patients with $\mathrm{Ph}+\mathrm{AML}$ and CML-MBP treated at our institution with venetoclax and BCR-ABL1 TKI-based combinations.

\section{Treatment Regimens}

Dosing of agents was performed per institutional guidelines and practice. Venetoclax was dosed at $400 \mathrm{mg}$ daily with dose reductions in conjunction with CYP3A4 inhibitors, e.g., azole antifungals for infection prophylaxis or treatment. Reduction in the venetoclax treatment period from continuous to $<2$ weeks was allowed in cases of myelosuppression. TKI was administered concomitantly with venetoclax. Dasatinib was dosed at $100 \mathrm{mg}$ daily $(n=2)$ or $50 \mathrm{mg}$ daily $(n=2)$, bosutinib was dosed at $400 \mathrm{mg}$ daily, nilotinib was dosed at $200 \mathrm{mg}$ daily, and ponatinib was dosed daily at $30 \mathrm{mg}$ $(n=7), 15 \mathrm{mg}(n=2)$, or $45 \mathrm{mg}(n=1)$. Two patients with concomitant FLT3-ITD received gilteritinib 80 and $120 \mathrm{mg}$ daily, respectively. Decitabine $\left(20 \mathrm{mg} / \mathrm{m}^{2}\right)$ was administered for 5-10 days. The CLIA2 regimen consisted of cladribine $5 \mathrm{mg} / \mathrm{m}^{2}$ on days $1-5$, cytarabine $2,000 \mathrm{mg} / \mathrm{m}^{2}$ i.v. on days $1-5$, and idarubicin $10 \mathrm{mg} / \mathrm{m}^{2}$ i.v. on days $1-3$. The CIA regimen consisted of clofarabine 22.5 $\mathrm{mg} / \mathrm{m}^{2}$ i.v. on days $1-5$, idarubicin $6 \mathrm{mg} / \mathrm{m}^{2}$ i.v. on days $1-3$, and cytarabine $0.75 \mathrm{~g} / \mathrm{m}^{2}$ i.v. on days $1-5$. The FIA regimen consisted of fludarabine $30 \mathrm{mg} / \mathrm{m}^{2}$ i.v. on days $1-5$, idarubicin $6 \mathrm{mg} / \mathrm{m}^{2}$ i.v. on days $1-3$, and cytarabine $0.75 \mathrm{~g} / \mathrm{m}^{2}$ i.v. on days $1-5$. Cladribine with HiDAC regimen consisted of cladribine $5 \mathrm{mg} / \mathrm{m}^{2}$ and cytarabine $1,000 \mathrm{mg} / \mathrm{m}^{2}$ i.v. daily for days $1-3$. CPX-351 was administered at $44 \mathrm{U} / \mathrm{m}^{2}$ on days 1,3 , and 5 . Patients could proceed to allogeneic stem cell transplantation (allo-SCT) after achievement of a response if they were deemed fit for allo-SCT and a donor was available.

\section{Response Assessment}

The AML European LeukemiaNet 2017 criteria were used to determine response [17]. The BCR-ABL1 kinase domain mutation analysis done on CDNA with a nested polymerase chain reaction (PCR) method covered codons 221-500 [18]. Flow cytometry for minimal residual disease (MRD) testing had a sensitivity of $0.1 \%$ or higher [19]. Karyotype or fluorescence in situ hybridization were used to determine the proportion of $\mathrm{Ph}+$ metaphases in bone marrow aspiration samples prior to starting therapy. Conventional karyotype was used to assess cytogenetic response after therapy.

\section{Data Sharing}

At this time, we will not be able to share individual patient level data outside of our institution.

\section{Statistical Analysis}

OS was determined from the date of therapy start to the date of death or censoring at the last follow-up. Relapse-free survival (RFS) was determined from the date of achievement of response to relapse, or death, or censoring at the last follow-up. OS and RFS were calculated using the Kaplan-Meier method. Student's $t$ test was used to determine the association between continuous variables and response. GraphPad Prism v7.0 (GraphPad Software, San Diego, CA, USA) was used for statistical analyses.

\section{Results}

\section{Patients and Treatment}

Between July 2017 and May 2019, 16 patients with $\mathrm{Ph}+$ AML and CML-MBP were treated with venetoclax and BCR-ABL1 TKI-based regimens. A summary of baseline patient characteristics and prior therapies is shown in Table 1 . Seven patients had $\mathrm{Ph}+\mathrm{AML}$, and 9 patients had CML-MBP. The median age of the patients was 42 years 
Table 1. Baseline characteristics of patients with $\mathrm{Ph}+\mathrm{AML}$ and CML in myeloid blast phase (MBP)

\begin{tabular}{lcc}
\hline Patient characteristics & $\begin{array}{l}\text { Ph+ AML } \\
(n=7)\end{array}$ & $\begin{array}{l}\text { CML-MBP } \\
(n=9)\end{array}$ \\
\hline Age, years & $47[21-73]$ & $38[26-75]$ \\
ECOG performance status $0-2$ & $6(86)$ & $7(78)$ \\
WBC count, $\times 10^{9} / \mathrm{L}$ & $9.7[1.1-25.7]$ & $6.9[0.1-131.5]$ \\
Peripheral blasts, $\%$ & $52[1-73]$ & $14[2-88]$ \\
Bone marrow blasts, $\%$ & $52[10-78]$ & $64[2-79]$ \\
Ph+ metaphases, $\%$ & $5[0-100]$ & $100[86-100]$ \\
BCR-ABL1 PCR, $\%$ & $7.05[0.03-17.01]$ & $71.74[0.58-100]$ \\
Complex cytogenetics & $5(71)$ & $3 / 6(50)$ \\
Prior therapies & $5[2-6]$ & $4[2-8]$ \\
$\quad$ Prior TKI & $3(43)$ & $9(100)$ \\
$\quad$ Prior venetoclax & $1(14)$ & $0(0)$ \\
BCR-ABL1 kinase domain mutation & $0 / 4(0)$ & $3 / 8(38)$ \\
Response to prior therapy & & $4(44)$ \\
$\quad$ Refractory & $6(86)$ & $2(22)$ \\
$\quad$ Relapse & $1(14)$ & $3(33)$ \\
$\quad$ Transformation from CML-CP & $0(0)$ & \\
\hline
\end{tabular}

Results are expressed as $n$ (\%) or medians [ranges]; CP, chronic phase; BCR-ABL1 RT$\mathrm{PCR}$ reported as the percentage of BCR-ABL1 to ABL1 transcripts. See text for other abbreviations.
Table 2. Venetoclax-based regimens in patients with $\mathrm{Ph}+\mathrm{AML}$ and CML in myeloid blast phase (MBP)

\begin{tabular}{lll}
\hline Regimen & $\begin{array}{l}\text { Ph+ AML } \\
(n=7)\end{array}$ & $\begin{array}{l}\text { CML-MBP } \\
(n=9)\end{array}$ \\
\hline $\begin{array}{l}\text { Decitabine based } \\
\text { Intensive chemotherapy based }\end{array}$ & $4(57)$ & $5(56)$ \\
$\begin{array}{l}\text { BCR-ABL TKI } \\
\quad \text { Ponatinib }\end{array}$ & $3(43)$ & $4(44)$ \\
$\quad$ Dasatinib & $6(86)$ & $4(44)$ \\
$\quad$ Bosutinib & $1(14)$ & $3(33)$ \\
Nilotinib & $0(0)$ & $1(11)$ \\
\hline
\end{tabular}

Results are expressed as $n(\%)$. See text for other abbreviations.

(range 21-73), and 13 patients (81\%) had ECOG performance status $\leq 2$. Eight of 13 patients $(62 \%)$ with evaluable karyotype had complex cytogenetics, and 7 of 11 patients (64\%) with next-generation sequencing results had $\geq 1$ adverse risk mutation as defined by AML ELN 2017 criteria. Three out of 11 tested patients (27\%) had a TP53 mutation. Nine patients were refractory to prior therapy, 3 patients had relapsed, and 4 patients had transformed from CML CP while on TKI therapy. Three of 12 patients tested prior to starting venetoclax plus the TKI combination harbored an $A B L 1$ kinase domain mutation including $1 \mathrm{mu}-$ tation each of T315I, E255K, and E355G. Patients had re- ceived a median of 5 prior lines of therapies (range 2-8), and CML-MBP patients had received a median of 2 prior TKI (range 1-3). Four of the 7 AML patients had received prior BCR-ABL1 TKI, including dasatinib $(n=3)$ and ponatinib $(n=1)$. One patient with $\mathrm{Ph}+$ acute lymphoblastic leukemia had received prior venetoclax. A summary of treatment regimens is shown in Table 2. Nine patients (57\%) received decitabine-based regimens, and the others received intensive chemotherapy-based regimens. Ten patients $(63 \%)$ received ponatinib while the other patients received second-generation TKI.

\section{Efficacy}

Characteristics, treatment regimens, and outcomes of individual patients are shown in Table 3. Fifteen of the 16 patients were evaluable for response as 1 patient had early death due to pneumonia. The overall response rate (ORR) in 15 evaluable patients was 60\%. One patient (7\%) achieved complete response (CR), 6 (40\%) achieved $\mathrm{CR}$ with incomplete hematologic recovery (CRi), 1 patient $(7 \%)$ achieved a morphologic leukemia-free state (MLFS), and 1 patient (7\%) had a partial response of extramedullary disease on positron emission tomography (PET)/computed tomography (CT). Another patient had hypoplastic bone marrow but did not meet the formal response criterion. The ORR in $7 \mathrm{Ph}+\mathrm{AML}$ patients was $43 \%(3 / 7)$, and in 8 evaluable CML-MBP patients ORR 
Table 3. Characteristics and outcomes of individual patients with advanced Philadelphia chromosome-positive $(\mathrm{Ph}+) \mathrm{myeloid}$ leukemia treated with venetoclax and tyrosine kinase inhibitor (TKI)-based regimens

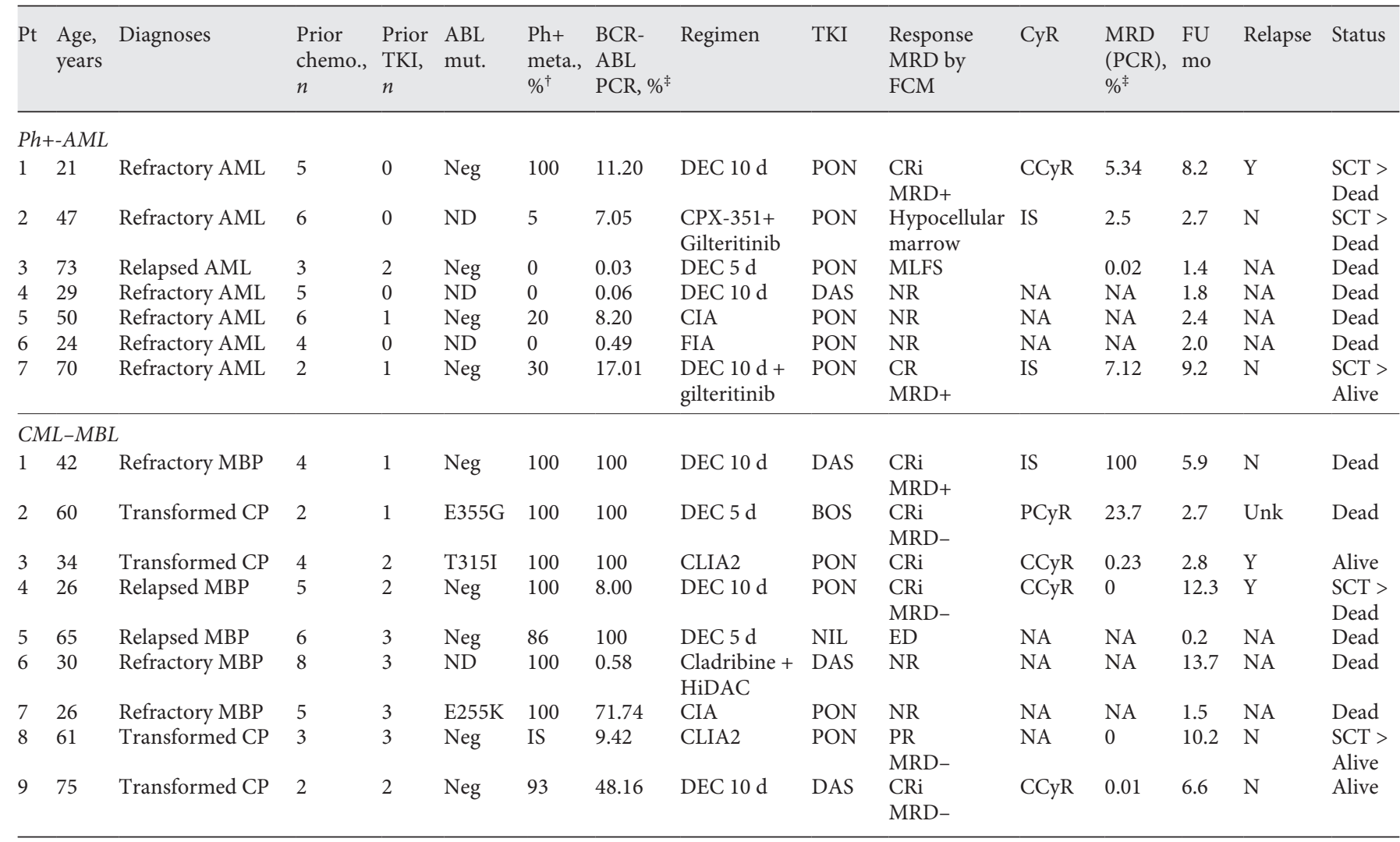

CML, chronic myeloid leukemia; MBP, myeloid blast phase; CP, chronic phase; DEC, decitabine; CLIA2, cladribine, idarubicin, cytarabine; HiDAC, high-dose cytarabine; CIA, clofarabine, idarubicin, cytarabine; PON, ponatinib; DAS, dasatinib; BOS, bosutinib; NIL, nilotinib; CR, complete remission; CRi, CR with incomplete hematologic recovery; PR, partial response of extramedullary disease on PET/CT; MRD, minimal residual disease; FCM, flow cytometry; ED, early death; NR, no response; NA, not applicable; Neg, negative; Unk, unknown, lost to follow-up; Y, yes; SCT, stem cell transplantation; FU, follow-up; mo, months; IS, insufficient sample; $\mathrm{ND}$, not done; $\mathrm{CyR}$, cytogenetic response; $\mathrm{CCyR}$, complete $\mathrm{CyR}=0 \% \mathrm{Ph}+$ metaphases; $\mathrm{PCyR}$, partial CyR = $1-35 \% \mathrm{Ph}+$ metaphases.

${ }^{\dagger}$ Metaphases harboring $\mathrm{t}(9 ; 21)$, reported as a percentage of karyotyped metaphases.

* BCR-ABL1 RT-PCR reported as the percentage of BCR-ABL1 to ABL1 transcripts.

was $75 \%(6 / 8)$. Median time to response in these 9 responding patients was 2.1 months (range $0.8-4.2$ ) with a median of 2 cycles of therapy to best response (range $1-4)$. Four patients proceeded to subsequent allo-SCT.

MRD negativity tested by flow cytometry was achieved in 3 of 7 (43\%) responding and evaluable patients. Median BCR-ABL1 PCR at response was $1.4 \%$ (range $0-23.7 \%)$ in 8 responding patients tested. Four patients achieved a complete cytogenetic response (CCyR) including 3 patients with CML-MBP and 1 patient with $\mathrm{Ph}+$ AML. Among 3 patients with TP53 mutation, 2 patients achieved a CRi and 1 patient achieved MLFS. Two patients with concomitant FLT3-ITD mutation treated with gilteritinib achieved CR with detectable MRD and hypocellular marrow, respectively.
Out of 10 patients who received ponatinib, 5 patients achieved CRi/MLFS, 1 patient achieved partial response on PET/CT, 1 patient had hypoplastic marrow with positive MRD, and 3 patients did not respond. Out of 6 patients who received second-generation TKI, 3 patients achieved CRi, 2 patients did not respond, and 1 patient had early death. Patients treated with ponatinib had a trend towards longer survival, with a median OS of 5.3 months compared to patients treated with second-generation TKI who had a median OS 3.6 months (hazard ratio $0.82,95 \%$ confidence interval $[\mathrm{CI}] 0.25-2.68, p=0.11$ ). Compared to nonresponders, patients achieving CR/CRi had a higher number of baseline $\mathrm{Ph}+$ metaphases (100 vs. $20 \%, p=0.06)$ and baseline BCR-ABL1 PCR (48.16 vs. $0.58 \%, p=0.05)$. The 2 patients in the AML cohort who 


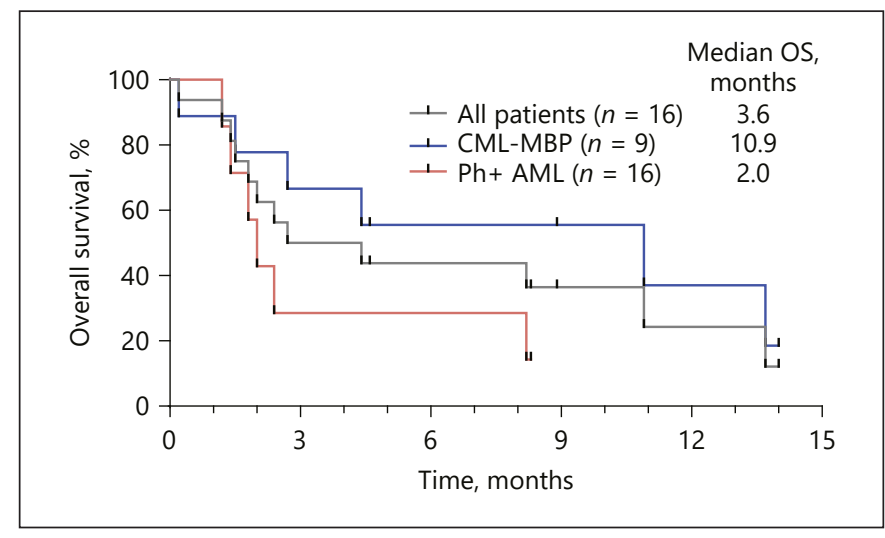

Fig. 1. Overall survival (OS) of patients with advanced Philadelphia chromosome-positive $(\mathrm{Ph}+)$ myeloid leukemias treated with venetoclax and $\mathrm{BCR}-\mathrm{ABL}$ tyrosine kinase inhibitor-based regimens.

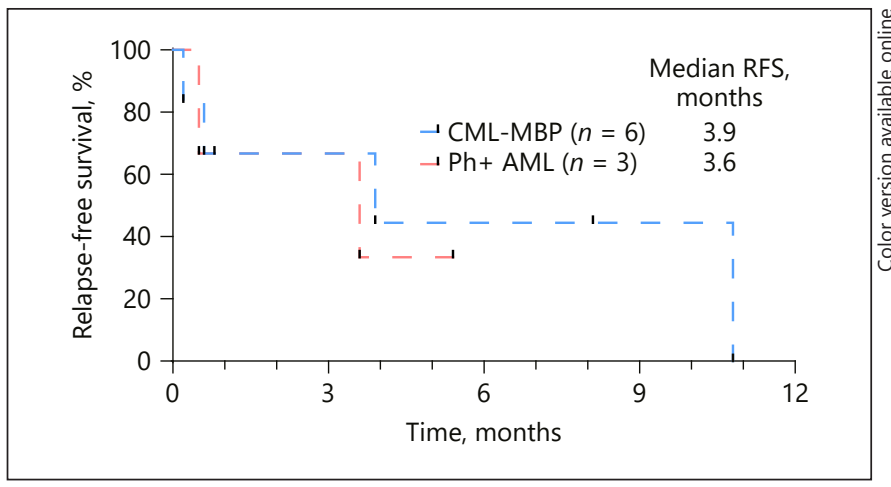

Fig. 2. Relapse-free survival (RFS) of patients with advanced Philadelphia chromosome-positive $(\mathrm{Ph}+)$ myeloid leukemias treated with venetoclax and BCR-ABL tyrosine kinase inhibitor-based regimens.

achieved CR/CRi were those who had the highest proportion of $\mathrm{Ph}+$ metaphases and highest BCR-ABL1 level by PCR. Both of these responders had $30 \%$ of higher $\mathrm{Ph}+$ metaphases on karyotype and had BCR-ABL1 PCR $>10 \%$.

Of patients who received decitabine-based regimens, 7 patients achieved CRi/MLFS, 1 patient did not respond, and 1 patient had early death. Out of 7 patients who received intensive chemotherapy-based regimens, 4 patients did not respond, 1 patient achieved CRi, 1 patient achieved partial response on PET/CT, and 1 patient had hypocellular marrow. Decitabine-based regimens yielded higher odds of achieving CRi/MLFS compared to intensive chemotherapy-based regimens (odds ratio 21.00, 95\% CI 1.50-293.25, $p=0.02$ ).

At a median follow-up of 14 months, 4 patients are alive, and 2 patients continue on therapy. Out of these 4 surviving patients, 3 patients received ponatinib, and 2 patients had undergone allo-SCT. There was 1 death within 30 days in a patient treated with a decitabine-based regimen due to pneumonia. There were no early deaths in patients treated with intensive chemotherapy-based regimens. Median OS for all patients was 3.6 months, for patients with CML-MBP it was 10.9 months, and for patients with $\mathrm{Ph}+\mathrm{AML}$ it was 2.0 months (Fig. 1). Median RFS for patients with CML-MBP was 3.9 months and for patients with Ph+ AML 3.6 months (Fig. 2). Patients treated with decitabine-based regimens had a median OS of 4.4 months, and for those receiving intensive chemotherapy-based regimens it was 2.4 months (hazard ratio $0.73,95 \%$ CI $0.24-2.26, p=0.56$ ). Reasons for treatment discontinuation in 14 patients included no response in 5 patients, SCT in 4 patients, relapse in 2 patients, loss to follow-up in 1 patient, sepsis in 1 patient, and early death in 1 patient.

\section{Discussion}

In this retrospective study, we showed feasibility and activity of venetoclax and TKI-based regimens in relapsed/refractory $\mathrm{Ph}+\mathrm{AML}$ and CML-MBP patients. This is the first report of clinical activity of this rational combination in a retrospective cohort of patients. In this heavily pretreated adverse risk group of patients with advanced $\mathrm{Ph}+$ myeloid leukemia, venetoclax and TKI-based regimens showed an ORR of $60 \%$ and median OS of 2 months in the $\mathrm{Ph}+\mathrm{AML}$ group and of 10.9 months in the CML-MBP group. The median OS of 10.9 months in this pretreated CML-MBP group was comparable to a previously reported median OS of 9-12 months in a cohort of patients with CML-MBP which included newly diagnosed and TKI-naive patients, both of which confer better outcomes compared to treated CML-MBP [2]. In contrast, within our cohort of patients with CML-MBP, the median number of prior TKI was 2 (range 1-3).

Dasatinib has shown single-agent activity in CML-MBP; however, the venetoclax -TKI combination may be better than single-agent dasatinib. With necessary precautions for cross-study comparisons, this combination of venetoclax with TKI-based regimens offered a median OS of 10.9 months in patients with CML-MBP compared to 7.9 months with single-agent dasatinib in a more favorable risk population [25]. Patients with CML-MBP in our cohort were more heavily pretreated and had received a median of 2 prior TKI and included relapsed/refractory patients while, in comparison, the dasatinib START-B trial only included patients who were imatinib resistant or intolerant. 
Ponatinib has shown favorable responses as single agent or in combination with nonchemotherapeutic agents in advanced $\mathrm{Ph}+$ leukemias and relapsed/refractory pre-B acute lymphoblastic leukemia [20, 21]. In our current report, 3 of the 4 surviving patients received ponatinib. We had previously reported that ponatinib showed the highest major hematologic and major molecular response rates of 67 and $27 \%$, respectively, compared to other TKI in patients with CML-BP [2]. Furthermore, preclinical studies have shown that ponatinib synergizes with venetoclax in $\mathrm{Ph}+$ acute lymphoblastic leukemia samples through induction of LYN-mediated pro-apoptotic BIM and decreasing anti-apoptotic MCL1, and potentially abrogating venetoclax resistance [22]. Hence, taken together, these data provide a strong rationale for combining venetoclax with ponatinib in this population. Decitabine-based regimens showed significantly higher response rates than intensive chemotherapy-based regimens, but OS was comparable. Given how venetoclax synergizes with both decitabine and intensive chemotherapy, either of these backbones may be a reasonable option for such patients depending on older versus younger age, and "fitness" for intensive therapy.

Despite limited numbers, there was a trend towards higher clonal burden of $\mathrm{Ph}+$ metaphases and higher baseline BCR-ABL1 PCR among patients who achieved CR/ CRi compared to nonresponders. The only 2 patients in the AML cohort who achieved CR/CRi had 30\% or higher Ph+ metaphases and BCR-ABL1 PCR of $10 \%$ or higher. Response rates also appeared higher in CML-MBP, which is more strongly driven by BCR-ABL1 signaling than is $\mathrm{Ph}+\mathrm{AML}$, which often only contains a subclonal BCRABL1-driven population. This suggests that venetoclax and TKI-based combinations may be particularly useful in patients where the $\mathrm{Ph}+$ clone is a dominant clone without other major coexisting drivers. We speculate that minor $\mathrm{Ph}$ subclones may develop in advanced leukemia due to chromosomal instability, and TKI addition may not offer additional benefit in such cases when BCR-ABL is not the main driver.

This was a retrospective study with all inherent limitations thereof. We could not assess the impact of venetoclax addition compared to TKI alone or with chemotherapy. The heterogeneous patient population and treatment regimens limit broad-reaching conclusions from these data. Given the retrospective nature of this study, we could not provide assessment of treatment-emergent adverse events. Hence, we have opened a clinical trial to prospectively evaluate this combination of decitabine, venetoclax, and ponatinib in Ph+ AML and CML-MBP (NCT04188405).

\section{Conclusion}

Venetoclax and TKI-based combination regimens are a feasible approach to treat advanced $\mathrm{Ph}+$ myeloid leukemias. Patients with CML-MBP may stand to benefit particularly from this combination due to BCR-ABL1 being the predominant driver. Previously published preclinical data of synergy and clinical activity reported here warrant further evaluation of such combinations in these poorrisk populations.

\section{Statement of Ethics}

This work complies with the guidelines for human studies and was conducted ethically in accordance with the World Medical Association Declaration of Helsinki. Patients signed a written informed consent before receiving the treatments described here, but requirement of informed consent for this study was waived due to the retrospective chart review nature of this study This study was approved by the Institutional Review Board and received ethics committee clearance..

\section{Disclosure Statement}

A.M.: research funding from Celgene Corporation; F.R.: research funding from Amgen, Bristol-Myers Squibb, Merck, Seattle Genetics, Sunesis Pharmaceuticals, and honoraria from Amgen, Pfizer, Seattle Genetics, Sunesis Pharmaceuticals; consulting or advisory role for Amgen, Seattle Genetics, Sunesis Pharmaceuticals; J.E.C.: research funding from Ambit BioSciences, ARIAD, Arog, Astellas Pharma, AstraZeneca, Bristol-Myers Squibb, Celator, Celgene, Novartis, Pfizer, Sanofi, Sun Pharma, Teva; consultant for Ambit BioSciences, ARIAD, Astellas Pharma, BiolineRx, BristolMyers Squibb, Novartis; Pfizer; E.J.J.: consultancy/research funding from Takeda, BMS, Adaptive, Amgen, AbbVie, Pfizer, Cyclacel; K.S.: Otsuka (honoraria) and Pfizer (consultancy); N.G.D.: consultancy from Sunesis Pharmaceuticals, Karyopharm, Pfizer, Bristol-Myers Squibb, Novartis Pharmaceuticals, Otsuka America Pharmaceutical, Jazz; research funding from Karyopharm, Immunogen, Pfizer, Incyte, Bristol-Myers Squibb, Daiichi-Sankyo, Kiromic; and honoraria from Incyte; T.M.K.: none, M.Y.K.: Calithera (research funding); Stemline Therapeutics (consultancy, honoraria, and research funding); Forty-Seven (consultancy and honoraria); Eli Lilly (research funding); AbbVie (consultancy, honoraria, and research funding); Cellectis (research funding); Amgen (consultancy and honoraria); Hoffman La-Roche (consultancy, honoraria, and research funding); Genentech (honoraria and research funding); Ascentage (research funding); Kisoji (consultancy and honoraria); Reata Pharmaceuticals (equity ownership and patents \& royalties); Ablynx (research funding); Astra Zeneca (research funding); and Agios (research funding); G.B.: research funding from AbbVie, Incyte, and Janssen; C.D.D.: honoraria and research funding from AbbVie, Agios, Novartis, Celgene, and Daiichi-Sankyo; M.F., L.M., K.N., S.P., and K.M.: no conflicts of interest; H.M.K.: research funding from Ariad, Astex, BMS, Cyclacel, Daiichi-Sankyo, Jazz, Novartis, Pfizer (also honoraria), Im- 
munogen (also honoraria), and honoraria from Actinium and Takeda; N.J.S.: Takeda Oncology (consultancy and research funding); AstraZeneca (consultancy); and Amgen (honoraria).

\section{Funding Sources}

This study was supported in part by the MD Anderson Cancer Center Support Grant CA016672 from the National Cancer Institute. N.J.S. is supported by the K12 Paul Calabresi Clinical Oncology Scholar Award and the American Society of Hematology Junior Faculty Scholar Award in Clinical Research.

\section{Author Contributions}

Conception and design: N.J.S., A.M., M.F., and S.P.; administrative support: H.M.K. and N.J.S.; provision of study materials or patients: F.R., J.E.C., E.J.J., K.S., K.M., N.G.D., M.Y.K., L.M., G.B., C.D.D., K.N., H.M.K., and N.J.S.; collection and assembly of data: A.M., M.F., S.P., K.S., and N.J.S.; data analysis and interpretation: A.M., M.F., and N.J.S.; manuscript writing: A.M., M.F., and N.J.S.; and critical revision for important intellectual content: all authors. All authors reviewed and approved the final version of the manuscript.

\section{References}

1 Short NJ, Rytting ME, Cortes JE. Acute myeloid leukaemia. Lancet. 2018 Aug;392 (10147):593-606

2 Jain P, Kantarjian HM, Ghorab A, Sasaki K, Jabbour EJ, Nogueras Gonzalez G, et al. Prognostic factors and survival outcomes in patients with chronic myeloid leukemia in blast phase in the tyrosine kinase inhibitor era: cohort study of 477 patients. Cancer. 2017 Nov; 123(22):4391-402.

3 Deschler B, Lübbert M. Acute myeloid leukemia: epidemiology and etiology. Cancer. 2006 Nov;107(9):2099-107.

4 Söderlund S, Dahlén T, Sandin F, OlssonStrömberg U, Creignou M, Dreimane A, et al. Advanced phase chronic myeloid leukaemia $(\mathrm{CML})$ in the tyrosine kinase inhibitor era - a report from the Swedish CML register. Eur J Haematol. 2017;98(1):57-66.

5 Soupir CP, Vergilio JA, Dal Cin P, Muzikansky A, Kantarjian H, Jones D, et al. Philadelphia chromosome-positive acute myeloid leukemia: a rare aggressive leukemia with clinicopathologic features distinct from chronic myeloid leukemia in myeloid blast crisis. Am J Clin Pathol. 2007 Apr;127(4): 642-50.

6 Keung YK, Beaty M, Powell BL, Molnar I, Buss D, Pettenati M. Philadelphia chromosome positive myelodysplastic syndrome and acute myeloid leukemia-retrospective study and review of literature. Leuk Res. 2004 Jun; 28(6):579-86.

7 Aoki J, Kakihana K, Kobayashi T, Hirashima $\mathrm{Y}$, Akiyama H, Ohashi K, et al. Tyrosine kinase inhibitor therapy for acute myeloid leukemia with late-appearing Philadelphia chromosome. Leuk Res. 2012 Jan;36(1):e41-2.

8 DeFilipp Z, Khoury HJ. Management of advanced-phase chronic myeloid leukemia. Curr Hematol Malig Rep. 2015 Jun;10(2): 173-81.

9 Apperley JF. Chronic myeloid leukaemia. Lancet. 2015 Apr;385(9976):1447-59.
10 Pan R, Hogdal LJ, Benito JM, Bucci D, Han L, Borthakur G, et al. Selective BCL-2 inhibition by ABT-199 causes on-target cell death in acute myeloid leukemia. Cancer Discov. 2014 Mar;4(3):362-75.

11 Gibson CJ, Davids MS. BCL-2 Antagonism to Target the Intrinsic Mitochondrial Pathway of Apoptosis. Clin Cancer Res. 2015 Nov; 21(22):5021-9.

12 Horita M, Andreu EJ, Benito A, Arbona C, Sanz C, Benet I, et al. Blockade of the Bcr-Abl kinase activity induces apoptosis of chronic myelogenous leukemia cells by suppressing signal transducer and activator of transcription 5-dependent expression of Bcl-xL. J Exp Med. 2000 Mar;191(6):977-84.

13 Goff DJ, Court Recart A, Sadarangani A, Chun HJ, Barrett CL, Krajewska M, et al. A Pan-BCL2 inhibitor renders bone-marrowresident human leukemia stem cells sensitive to tyrosine kinase inhibition. Cell Stem Cell. 2013 Mar; 12(3):316-28.

14 Quintás-Cardama A, Qiu YH, Post SM, Zhang Y, Creighton CJ, Cortes J, et al. Reverse phase protein array profiling reveals distinct proteomic signatures associated with chronic myeloid leukemia progression and with chronic phase in the CD34-positive compartment. Cancer. 2012 Nov;118(21):5283-92.

15 Carter BZ, Mak PY, Mu H, et al. Combined targeting of BCL-2 and BCR-ABL tyrosine kinase eradicates chronic myeloid leukemia stem cells. Sci Transl Med. 2016 Sep;8(355): $355 \mathrm{ra} 117$.

16 Okabe S, Tauchi T, Tanaka Y, Ohyashiki K. Anti-Leukemic Effects of Venetoclax on Philadelphia Chromosome Positive Leukemia Cells. Blood. 2016 Dec;128(22):5428.

17 Döhner H, Estey E, Grimwade D, Amadori S, Appelbaum FR, Büchner T, et al. Diagnosis and management of AML in adults: 2017 ELN recommendations from an international expert panel. Blood. 2017 Jan;129(4):424-47.

18 Luthra R, Sanchez-Vega B, Medeiros LJ. TaqMan RT-PCR assay coupled with capillary electrophoresis for quantification and identification of bcr-abl transcript type. Mod Pathol. 2004 Jan;17(1):96-103.
$19 \mathrm{Xu}$ J, Jorgensen JL, Wang SA. How Do We Use Multicolor Flow Cytometry to Detect Minimal Residual Disease in Acute Myeloid Leukemia? Clin Lab Med. 2017 Dec;37(4): 787-802.

20 Cortes JE, Kim DW, Pinilla-Ibarz J, le Coutre P, Paquette R, Chuah C, et al.; PACE Investigators. A phase 2 trial of ponatinib in Philadelphia chromosome-positive leukemias. N Engl J Med. 2013 Nov;369(19):1783-96.

21 Assi R, Kantarjian H, Short NJ, Daver N, Takahashi K, Garcia-Manero G, et al. Safety and Efficacy of Blinatumomab in Combination with a Tyrosine Kinase Inhibitor for the Treatment of Relapsed Philadelphia Chromosome-positive Leukemia. Clin Lymphoma Myeloma Leuk. 2017 Dec;17(12):897-901.

22 Leonard JT, Rowley JS, Eide CA, Traer E, Hayes-Lattin B, Loriaux $M$, et al. Targeting BCL-2 and ABL/LYN in Philadelphia chromosome-positive acute lymphoblastic leukemia. Sci Transl Med. 2016 Aug;8(354): $354 \mathrm{ra} 114$

23 Maiti A, DiNardo CD, Rausch CR, et al. TenDay Decitabine with Venetoclax (DEC10VEN) in Acute Myeloid Leukemia: Updated Results of a Phase II Trial. Blood. 2019; 134(Supplement_1):2637.

24 Aboudalle I, Konopleva MY, Kadia TM, et al. A Phase Ib/II Study of the BCL-2 Inhibitor Venetoclax in Combination with Standard Intensive AML Induction/Consolidation Therapy with FLAG-IDA in Patients with Newly Diagnosed or Relapsed/Refractory AML. Blood. 2019;134(Supplement_1):176176

25 Saglio G, Hochhaus A, Goh YT, Masszi T, Pasquini R, Maloisel F, et al. Dasatinib in imatinib-resistant or imatinib-intolerant chronic myeloid leukemia in blast phase after 2 years of follow-up in a phase 3 study: efficacy and tolerability of 140 milligrams once daily and 70 milligrams twice daily. Cancer. 2010 Aug; 116(16):3852-61.
Venetoclax and TKI-Based Regimens in Advanced $\mathrm{Ph}+$ Leukemias
Acta Haematol 2020;143:567-573 DOI: $10.1159 / 000506346$ 